\title{
Inter-cell interference alignment in multi cell multiuser channels
}

\author{
Duckdong Hwang ${ }^{1,2 a)}$ \\ ${ }^{1}$ Samsung Electronics Co. \\ ${ }^{2}$ STE Team, DMC Research Center \\ a)duckdong.hwang@samsung.com
}

\begin{abstract}
We propose an interference alignment scheme for the multi cell multiuser interference channel, where $M$ antennas are used at the base stations and $N$ antennas are used at user terminals. The large number of interference vectors from other cells are aligned into interference alignment planes at the other cell base stations. After the alignment, $L$ cells support up to $L(\lfloor N /(L-1)\rfloor-1)$ user streams when $1 \leq\lfloor N /(L-1)\rfloor \leq M$.
\end{abstract}

Keywords: interference alignment, multi cell multiuser channel Classification: Wireless circuits and devices

\section{References}

[1] V. R. Cadambe and S. A. Jafar, "Interference Alignment and Degrees of Freedom of the K-User Interfernce Channel," IEEE Trans. Inf. Theory, vol. 54, no. 8, pp. 3425-3441, Aug. 2008.

[2] S. A. Jafar and M. J. Fakhereddin, "Degrees of Freedom for the MIMO Interference Channel," IEEE Trans. Inf. Theory, vol. 53, no. 7, pp. 26372642, July 2007.

[3] T. Gou and S. A. Jafar, "Degrees of Freedom of the K User $M \times N$ MIMO Interference Channel," IEEE Trans. Inf. Theory, vol. 56, no. 12, pp. 6040-6057, Dec. 2010.

[4] S. A. Jafar and S. Shamai, "Degrees of Freedom Region of the MIMO X Channel," IEEE Trans. Inf. Theory, vol. 54, no. 1, pp. 151-170, Jan. 2008.

[5] C. Suh, D. Tse, and M. Ho, "Downlink Interference Alignment," Proc. IEEE Globecom, Dec. 2010.

[6] T. Kim, D. Love, B. Clerckx, and D. Hwang, "Spatial Degree of Freedom of the Multicell MIMO Multiple Access Channel," Proc. IEEE Globecom, Dec. 2010.

[7] C. Suh and D. Tse, Proc. Allerton Conference on Communication, Control, and Computing, Sept. 2008.

\section{Introduction}

Competing wireless links can share the wireless resources like time or frequency through the Interference Alignment (IA) $[1,2,3,4]$ and enhance the 
usage of the spectrum. IA in two cell interfering multiuser system with multiple antenna nodes is considered in $[5,6]$. In [5], authors present an IA scheme, where $2(M-1)$ user streams can be supported when $M$ antennas are used at both the Base Station (BS) and user terminal. Aligning the interference vectors into the null spaces of the interference channels, the same number of user streams can be served with $M-1$ user terminal antenna elements ( $M$ antennas at BS) by the scheme appeared in [6].

In practical cellular systems, more than two cells interfere with each other. Hence, the IA schemes in $[5,6]$ are not much useful in real world interfering cells. Moreover, the number of user node antennas $N$ is fixed to $M$ in [5] and to $M-1$ in [6]. The IA scheme in this letter works for general $L$ cell MIMO interference channel with arbitrary $M$ and $N$. The large number of interference channels from multiple cells are aligned into a space spanned by the column vectors of the interference alignment plane matrix.

After the system model is introduced in Section 2, the IA scheme in $L$ cell is presented with some simulation results in Section 3. Section 4 concludes the letter.

\section{System model}

There are $L$ neighboring base stations where each BS serves $K(\geq 1)$ users. $M$ antennas are used at each BS and $N$ antennas are used at each user. Our derivation is based on the Multiple Access Channel (MAC). Using the updown duality given in [7], the proposed IA scheme can be applied in Broadcast Channel (BC) case as well. The signals from the $L K$ users reach the $L$ BS's. Since only $K$ user signals are associated with the $\ell$-th BS, there are $(L-1) K$ inter-cell interference signals for that BS. The received signal for the $\ell$-th BS is

$$
\mathbf{y}_{\ell}=\sum_{k=1}^{K} \mathbf{H}_{\ell, k} \mathbf{w}_{\ell, k} x_{\ell, k}+\sum_{l \neq \ell}^{L} \sum_{k=1}^{K} \mathbf{G}_{\ell, l, k} \mathbf{w}_{l, k} x_{l, k}+\mathbf{n}_{\ell},
$$

where $\mathbf{H}_{\ell, k}$ is the $M \times N$ channel matrix from the user $k$ in the $\ell$-th cell and $\mathbf{G}_{\ell, l, k}$ is the $M \times N$ interference channel matrix from the user $k$ in the near-by cell $l$. We assume that the elements of the channel matrices are independent and identically distributed (i.i.d.). A $N \times 1$ vector $\mathbf{w}_{l, k}$ with unit power is used to pre-code the message signal $x_{l, k}$ for the $k$-th user in the $l$-th cell. The power of the signal $x_{l, k}$ is $P /(L K)$, where $P$ is the total power used in $L$ cells. The $M \times 1$ vector $\mathbf{n}_{\ell}$ is the complex white Gaussian noise. Note that the number of interference terms in the second summation of (1) depends on $L$ and the number of users per cell, which makes the interference alignment hard if $L$ is large.

Let the columns of $M \times(L-1) K$ matrix $\mathbf{G}_{\ell}$ be the interference vectors $\left(\mathbf{G}_{\ell, l, k} \mathbf{w}_{l, k}\right.$, for all $l$ and $\left.k\right)$ from the other cell users. If the condition

$$
\operatorname{rank}\left(\mathbf{G}_{\ell}\right)=J, 1 \leq J \leq M
$$

is satisfied for all $l$, the $\ell$-th cell BS has $M-J$ interference free dimensions. Let the $M \times J$ matrix $\mathbf{P}_{\ell}$ be the alignment plane matrix of the $\ell$-th cell. If the 
interference vectors toward the $\ell$-th cell BS (the columns of matrix $\mathbf{G}_{\ell}$ ) are aligned such that they are spanned by the columns of $\mathbf{P}_{\ell}$, then the condition (2) is satisfied. If $K \leq(M-J)$, then we can find the $\ell$-th cell zero forcing (ZF) receiver to null out the rsulting interference. With the $\mathrm{ZF}$ receiver, we are given the following signal model.

$$
\mathbf{r}_{\ell}=\mathbf{F}_{Z F, \ell} \mathbf{y}_{\ell}
$$

where $K \times M$ the ZF matrix $\mathbf{F}_{Z F, \ell}$ is composed of the top $K$ rows of the pseudo inverse of $\mathbf{H}_{\ell}$. Here, $\mathbf{H}_{\ell}=\left[\mathbf{H}_{\ell, 1} \mathbf{w}_{\ell, 1}, \ldots, \mathbf{H}_{\ell, K} \mathbf{w}_{\ell, K}, \mathbf{p}_{\ell, 1}, \ldots, \mathbf{p}_{\ell, J}\right]$, and $\mathbf{p}_{\ell, j}$ is the $j$-th column of $\mathbf{P}_{\ell}$.

\section{The IA scheme and simulation results}

Let us consider the 3 cell case first. First, we check the condition to satisfy the condition in (2) for the signals $\mathbf{y}_{l}$ at the second cell and the third cell respectively. For the $k$-th user in the first cell, the pre-coder $\mathbf{w}_{1, k}$ should satisfy the two equations in (4) at the same time.

$$
\mathbf{P}_{2}^{\perp} \mathbf{G}_{2,1, k} \mathbf{w}_{1, k}=\mathbf{0}_{(M-J)}, \mathbf{P}_{3}^{\perp} \mathbf{G}_{3,1, k} \mathbf{w}_{1, k}=\mathbf{0}_{(M-J)} .
$$

Here, the $(M-J) \times M$ matrix $\mathbf{P}_{l}^{\perp}$ is the projection matrix orthogonal to the alignment plane matrix $\mathbf{P}_{l}$ and $\mathbf{0}_{(M-J)}$ is the $(M-J) \times 1$ zero vector. If (4) is satisfied for all $k=1, \ldots, K$, then (2) is satisfied for the 2 nd and the 3rd cells. Let us consider the $2(M-J) \times N$ matrix $\left[\begin{array}{c}\mathbf{P}_{2}^{\perp} \mathbf{G}_{2,1, k} \\ \mathbf{P}_{3}^{\perp} \mathbf{G}_{2,1, k}\end{array}\right]$. Since the elements of the channel matrices are i.i.d., the condition $2(M-J)<N$ is necessary for us to find a $\mathbf{w}_{1, k}$ that satisfies (4). The minimum $J$ required is $M-\lfloor N / 2\rfloor+1$, where $\lfloor\cdot\rfloor$ is the integer floor function. Since $J$ is in the range $1 \leq J \leq M$, we need the condition $1 \leq\lfloor N / 2\rfloor \leq M$. The same inequality is acquired if we check the conditions $\mathbf{w}_{2, k}$ and $\mathbf{w}_{2, k}$ should satisfy to meet (4). The first cell can serve up to $M-J=\lfloor N / 2\rfloor-1$ user streams without the other cell interference. In three cells, the maximum number of user streams served is $3(\lfloor N / 2\rfloor-1)$.

We can generalize the result into the $L$ cell interference channel network. If we follow the similar steps in the three cell case, $\mathbf{w}_{1, k}$ should satisfy the following conditions.

$$
\mathbf{P}_{l}^{\perp} \mathbf{G}_{l, 1, k} \mathbf{w}_{1, k}=\mathbf{0}_{(M-J)}, \text { for } l=2, \ldots, L .
$$

Similarly, the condition $(L-1)(M-J)<N$ is necessary to find $\mathbf{w}_{1, k}$ satisfying (5). Again, the minimum $J$ required is $M-\lfloor N /(L-1)\rfloor+1$ and the $L$ cells can serve up to $L(\lfloor N /(L-1)\rfloor-1)$ user streams when $1 \leq\lfloor N /(L-1)\rfloor \leq M$. In general, large $N$ is necessary as the number of cells $(L)$ becomes large to align the interference vectors toward the set of alignment planes.

We run simulations with some parameter sets and the sum rate results of the proposed IA scheme are plotted in Fig. 1. The sum rates of the $L K$ users in $L$ cells are calculated from the Shannon's capacity expressions ${ }^{1}$. From

\footnotetext{
${ }^{1}$ Expressed as $\log _{2}\left(1+S I N R_{l, k}\right)$ when $S I N R_{l, k}$ is the signal to noise power ratio of the $k$-th user in the $l$-th cell.
} 
the slopes of the sum rates, it is confirmed that the proposed IA scheme achieves the predicted degrees of freedom (user streams) with the inter-cell interference efficiently aligned and suppressed by the ZF receivers.

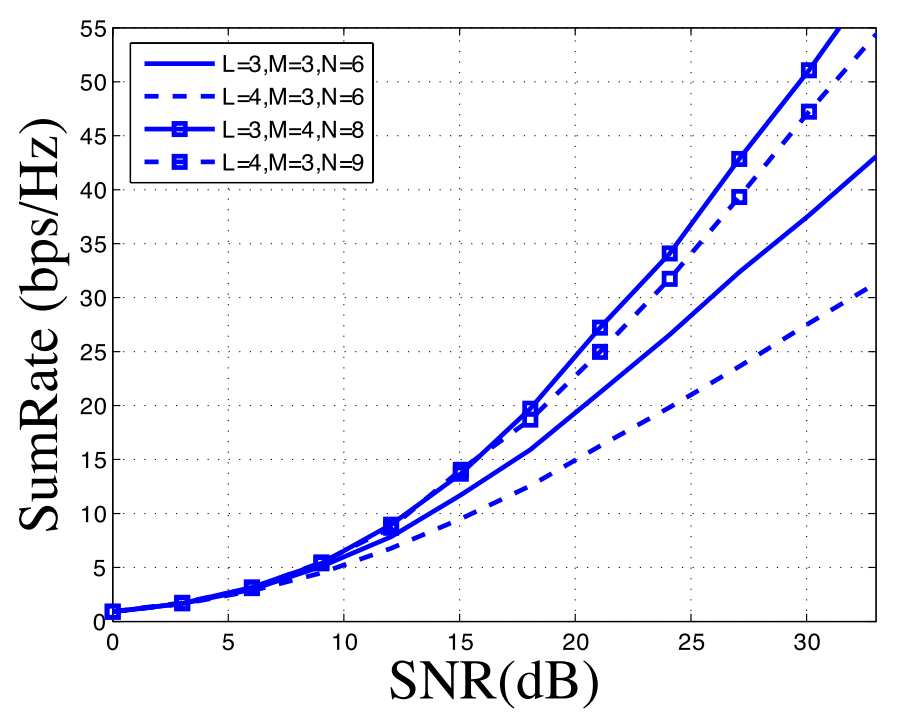

Fig. 1. Sum rate curves of the proposed IA scheme with different parameter sets. Solid lines are for $L=3$ and dashed lines are for $L=4$.

\section{Conclusion}

We propose an interference alignment scheme for multi cell multiuser interference channel with multiple antennas. We show that large number of inter cell interferences can be efficiently handled and aligned. Simulation results confirm that the proposed degrees of freedom values are achieved by the proposed alignment and suppression scheme. 